\title{
A MULTIPLE-BEAM INJECTOR FOR HEAVY ION INERTIAL FUSION*
}

\author{
J. W. Kwan, O. A. Anderson, D.N. Beck, F. M. Bieniosek, C. F. Chan, A. Faltens, E. Henestroza, \\ S. A. MacLaren, P. A. Seidl, LBNL, Berkeley, CA; L. Ahle, D. P. Grote, E. Halaxa, \\ C. T. Sangster, LLNL, Livermore, CA; W. B. Herrmannsfeldt, SLAC, Stanford, CA
}

\section{Abstract}

In a typical heavy ion fusion driver design, the induction linac requires a multiple beam injector. We present a conceptual design for an 84-beam injector system with each beam channel carrying $0.5 \mathrm{~A}$ of beam current. Each channel starts with a $10-\mathrm{cm}$ diameter surface ionization source followed by a 1.7 MV electrostatic Einzel lens-type preaccelerator and an electrostatic quadrupole (ESQ) matching section. The preaccelerator and matching section are $0.7 \mathrm{~m}$ and $5.0 \mathrm{~m}$ long respectively. The array has an overall diameter of $3.0 \mathrm{~m}$ at the ion source end and $1.0 \mathrm{~m}$ diameter at the exit.

\section{INTRODUCTION}

For heavy ion driven inertial fusion, the primary approach in the US is the induction linac [1]. Typical beam energy delivered to the target is $\sim 5 \mathrm{MJ}$ with a pulse length of $10 \mathrm{~ns}$ and a particle kinetic energy $\sim 5-10 \mathrm{GeV}$. Thus the total beam charge is $\sim 0.5-1.0 \mathrm{mC}$. This beam charge is supplied by the injector, although the pulse length here is typically less than $20 \mu \mathrm{s}$ long, based on the volt-sec limitation of the induction cores. The total beam current output from the injector is therefore expected to be in the order of $50 \mathrm{~A}$. Typical ions of interest are those of $\mathrm{Bi}$, $\mathrm{Pb}, \mathrm{Hg}, \mathrm{Cs}, \mathrm{Xe}, \mathrm{Rb}, \mathrm{K}, \mathrm{Ar}$, and $\mathrm{Ne}$, generally singly charged, but higher charge states are also of interest in some driver designs. Some lighter ions such as K, Ar and $\mathrm{Ne}$ are included because at the early stages of driver development they provide an opportunity to do experiments at driver-scale ion velocities on a medium length accelerator facility.

There are two main reasons for producing the $50 \mathrm{~A}$ beam current using many beams of smaller current (e.g., less than $1 \mathrm{~A}$ each). First, multiple beams provide better target illumination symmetry. This is an important requirement for high gain direct-drive targets. Second, heavy ion beams have significant space-charge effects, so the current per beam is limited by the focusing capabilities of the beam extraction and transport systems. Our goal is to design an injector that will produce an array of parallel beams feeding into the channels of a multiplebeam induction linac.

* This work is supported by the Office of Fusion Energy Science, US DOE under contract No. DE-AC03-76SF00098 (LBNL) and W-7405-ENG-48 (LLNL).

Email: jwkwan@lbl.gov

\section{OPTIMIZING CURRENT DENSITY}

Beam focussing in an induction linac can be achieved by using either electrostatic quadrupoles (ESQ) or magnetic quadrupoles. At the front end where the ion velocity is still low, ESQs are more effective and economical than magnetic quadrupoles. By considering the line charge density in ESQ beam transport, one can determine the average beam current density $\mathrm{J}_{\mathrm{ave}}$ for an array of beams [2]. For a given total beam current, higher $\mathrm{J}_{\text {ave }}$ means smaller induction cores and therefore lower cost.

Since the voltage spark down threshold scales favorably with small ESQs, the optimum $\mathrm{J}_{\text {ave occurs at a point }}$ when the ESQ size cannot be further reduced in order to allow enough beam clearance for possible electrode alignment and beam steering errors. For example, in the ILSE/Elise design [2], the ESQs at the beginning of the induction linac ( $2 \mathrm{MeV}$ energy) have a bore radius of 2.33 $\mathrm{cm}$ which corresponds to a conservative clearance of 1.0 $\mathrm{cm}$. In this case, the required beam current in each channel is $0.8 \mathrm{~A}$ of $\mathrm{K}^{+}$; with an average beam radius of $1.0 \mathrm{~cm}$, and a current density of $254 \mathrm{~mA} / \mathrm{cm}^{2}$ in the channel. Since the ESQ electrodes are round rods with a radius 8/7 times the bore radius, the current density averaged over the entire array is only $16.5 \mathrm{~mA} / \mathrm{cm}^{2}$. A more aggressive design using smaller clearance will result in less beam current per channel but a higher $\mathrm{J}_{\text {ave }}$.

At first look, it appears that the injector will require an array of ion sources that can provide the above $\mathrm{J}_{\mathrm{ave}}$. However, the actual critical component is the preaccelerator ion gun, which is responsible for accelerating the beam to a suitable energy and focussing the beam envelope before injecting into the ESQ matching section. In our earlier attempt to design a $2 \mathrm{MV}$ ion gun using the conventional Pierce type column, we found that the beam current was well below $0.5 \mathrm{~A}$ at the sparkdown limit. There are two ways to deal with this problem: either reduce the gun to a simple Pierce diode by starting the ESQ section at a lower energy or optimize the gun performance (by using an accel-decel scheme) to reach an acceptable current limit. The previous ILSE/Elise injector was constructed according to the first method. Its $750 \mathrm{kV}$ Pierce diode extracted $0.8 \mathrm{~A} \mathrm{~K}^{+}$. Since the ESQ dimensions scale inversely with beam energy (for the same current), $\mathrm{J}_{\text {ave }}$ at the ESQ entrance was reduced to $\sim 1.0 \mathrm{~mA} / \mathrm{cm}^{2}$. In the following sections, we present an injector design using the second method. It is based on a 1.7 MV ion gun capable of transporting $0.5 \mathrm{~A} \mathrm{~K}^{+}$current. 


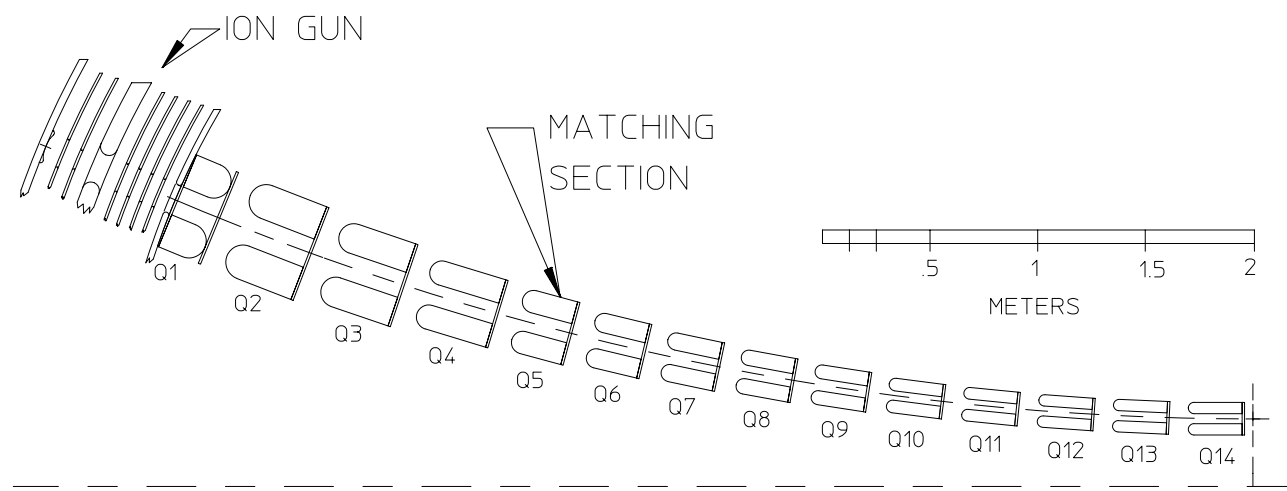

Figure 1: Schematic diagram of a single beamline at the edge of the injector array.

\section{THE PREACCELERATOR AND ESQ MATCHING SECTION}

According to our past experience in building the ILSE single beam injector [3], an injector that allows each beam to occupy an independent focussing channel and vacuum housing will be unacceptably large and costly. Therefore it is essential to arrange multiple beams in an array by sharing common electrodes, supporting structures, power supplies and vacuum systems.

Each beamline of the multiple-beam injector array consists of a gun and a matching section. Figure 1 shows a schematic diagram of a single beamline at the edge of the array. The gun is composed of a $200 \mathrm{kV}$ diode extracting $500 \mathrm{~mA}$ of $\mathrm{K}^{+}$from a $10-\mathrm{cm}$ diameter source aperture, followed by an Einzel lens column that focuses and accelerates the beam to $1.7 \mathrm{MV}$. The peak electric field stress in the gun is kept below $100 \mathrm{kV} / \mathrm{cm}$ to avoid electrical breakdown. Under this restriction, a standard Pierce-column (beam energy increases monotonically) cannot produce a converging beam. Our solution is to use Einzel lens (accel-decel) to provide extra beam focusing without exceeding the breakdown limit.

The matching section will simultaneously compress the beam envelopes and transform a round beam into an elliptical shape in order to match the alternating-gradient (AG) focusing lattice in the induction linac. As shown in Fig. 1, the ESQ electrodes in the matching section gradually decrease in size, thus a multiple beam array has the shape of a funnel. In order to avoid a sharp bend at the linac, the beam trajectories are deflected by a small angle at each ESQ module in the matching section. Eventually, the beams are brought together into parallel channels just before injecting into the downstream lattice.

In an ideal quadrupole, the transverse electric field gradient is constant within the aperture, thus an off-center beam will experience a combination of quadrupole and dipole fields; the dipole field will steer the beam centroid. In most parts of our design, the required beam displacement is small compared to the beam aperture. The beam envelope has a maximum excursion at the $2^{\text {nd }}$ quadrupole $(\mathrm{Q} 2)$, therefore the overall size of the array (and the required bending angle) is dictated by the required bore diameter at Q2. In the present case, the ion guns are straight and the ion sources are separated by $30.5 \mathrm{~cm}$. A surface ionization source can meet the specifications in beam current and low emittance (normalized edge $\varepsilon<1$ pimm-mr) [4]. As long as Q2 is dominating the size requirement, higher $\mathrm{J}_{\mathrm{ave}}$ at the ion source is not needed.

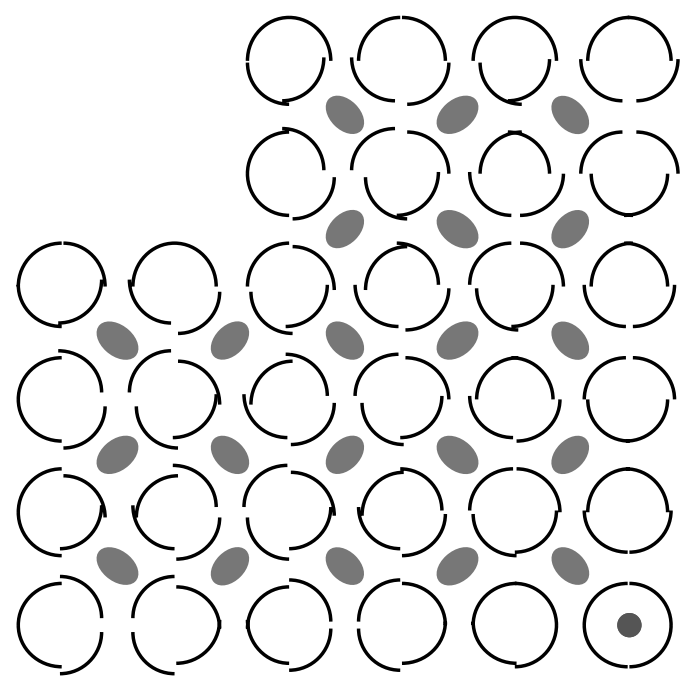

Figure 2: Cross section view of $1 / 4$ of an 84-beam array.

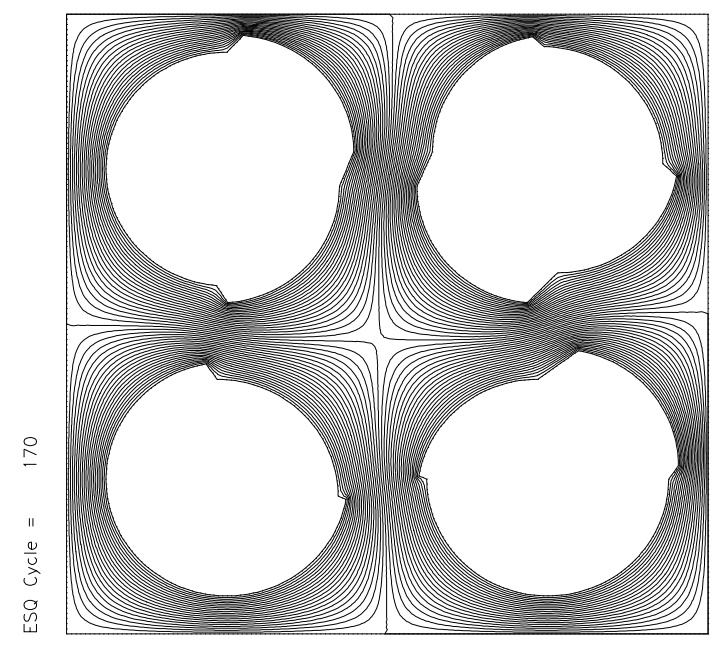

Figure 3: POISSON field calculation of a modified ESQ. 


\subsection{Multiple-beam array}

The beamline on the edge of the array (as shown in figure 1) has an angle of $333 \mathrm{mr}$. There is no bending at the first 2 quads where the beam envelopes are large. Q3 and Q4 each provides about $33 \mathrm{mr}$ bending, while the remaining angle is equally divided among Q5-Q14. In bending the beam, Q3 and Q4 are offset by $\sim 9 \mathrm{~mm}$ but the offset gradually reduces down to $1.5 \mathrm{~mm}$ at the final quad.

One advantage of packing multiple beams in an array is to share electrodes between beams. However the bending requirement complicates matters significantly because neighboring beams bend by different angles. In addition, there is a polarity change between adjacent quadrupoles such that the required offsets are in opposite directions. Figure 2 is a cross section view of $1 / 4$ of the array at the $4^{\text {th }}$ quadrupole plane. It shows how the electrode surfaces need to be altered (from a perfect circular pattern) in order to bend the beams through the desired arcs.

A simple ESQ geometry using round rods has a good field aperture of nearly $80 \%$ of the mechanical aperture. However here the ESQ electrodes are divided into 4 sections, each facing a given beamlet and with a fixed horizontal and vertical offset. Figure 3 shows the result from POISSON field calculation of the worse case (ESQ at the edge of the array). It confirms that aberrations are introduced by the distortions in the electric field structure. These aberrations reduce the effective aperture of the quadrupoles (possibly by as much as 50\%). Thus in order to avoid introducing emittance growth, we may consider lengthening the ESQ section or enlarging the ESQ array transversely.

Figure 4 is a drawing of an ESQ electrode. Typically the surfaces are at a slight angle, $\sim 2^{\circ}$ with respect to the electrode axis, in order to produce the funneling effect. The electrodes are hollow for minimizing weight. They must be fabricated and mounted onto a spherical support plate with a positioning tolerance of about $0.1 \mathrm{~mm}$. Based on preliminary price quotes, the most cost-effective way in machining these ESQ electrodes is to use wire electrical discharge machining (EDM). An initial bid for fabricating these ESQ electrodes was priced at $\$ 1500$ each.

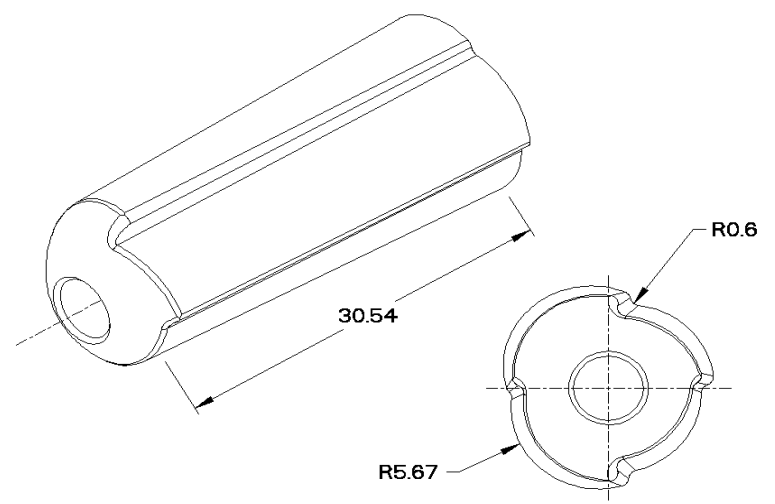

Figure 4: Drawing of a Q4 electrode near edge of array (nominal radius is $5.5 \mathrm{~cm}$ ).
One other way to funnel many beams without using offset electrodes is to slowly shrink the quadruples. In this case, one-half of the beams in the array at any given quadrupole plane will be bending in the wrong direction, but over any two successive quadrupole planes the net bending will be correct for all beams (similar to AG focusing). In order to avoid excessive space charge expansion in the z-direction, the matching section can not be arbitrary long. Preliminary simulation result indicated that a 50-m length is probably acceptable. At this point, it is not clear which method is more cost effective: a $50 \mathrm{~m}$ matching section with simple round rod electrodes or a $5 \mathrm{~m}$ matching section with specially shaped electrodes.

\subsection{Multi-aperture extraction and preacceleration}

Although the present design has already reduced the overall size of a multiple-beam injector compared with those in the previous designs using separate beamlines, the array is still very large at the source end. At present, we are developing an alternate concept that will produce a significantly smaller injector--one that possibly does not require a funnel-shaped geometry. The basic idea of this concept is to use miniature beamlets to overcome the beam transport problem mentioned in section 2. A multiaperture grid at the ion source can obtain high current density beamlets. Since the apertures are small, each beamlet will have a current of only a few $\mathrm{mA}$ and can be focused while accelerating to $\sim 1.7 \mathrm{MV}$. After preacceleration, the beamlets are merged together to form beams of 0.5 A each, ready for injecting into an ESQ channel. There will be emittance growth from beam merging, however the effect can be minimized by using a large number of beamlets and high grid transparency. Our preliminary estimation indicates that an injector system based on this principle is promising but requires further studies.

\section{SUMMARY}

In our conceptual design for an 84-beam injector system for HIF, the beamlines are arranged in an array that has the shape of a funnel. Each beamline consists of an ion source, a 1.7 MV ion gun and an ESQ matching section. Beam steering is done by displacing the ESQ electrodes. We are also continuing to explore alternate concepts that are either more compact or more cost effective.

\section{REFERENCES}

[1] R.O. Bangerter, Proc. of Inter. Symp. on Heavy Ion Inertial Fusion, Frascati, Italy, May 25-28, 1993, Nuovo Cimento 1445 (1993).

[2] J. W. Kwan, et al, Proc. of Inter. Symp. on Heavy Ion Inertial Fusion, Princeton, New Jersey, USA, Sept 6-9 (1995); Fusion Engineering and Design, 32-33, p299, (1996).

[3] S. Eylon, E. Henestroza, W.W. Chupp, and S. Yu, Proc. of Inter. Symp. on Heavy Ion Inertial Fusion, Frascati, Italy, May 25-28, 1993, Nuovo Cimento 1509 (1993).

[4] J. W. Kwan, W.W. Chupp, and S. Eylon, Proc. Particle Accelerator Conf., Vancouver, May, (1997). 